\title{
Pérennité et mutations en formation à distance à l'université Retour sur le vécu des vingt années d'existence du campus numérique FORSE
}

\section{Sustainability and changes in distance learning at the university: Review of twenty years of existence of the digital campus FORSE}

\section{Perennidad y mutaciones en formación a distancia en la universidad: Repasando los veinte años de existencia del campus digital FORSE}

Béatrice Verquin Savarieau, professeure

CIRNEF, Université de Rouen Normandie, France

beatrice.savarieau@univ-rouen.fr

Stéphane Simonian, professeur

Université Lumière Lyon 2, France

stephane.simonian@univ-lyon2.fr

Jacques Béziat, professeur

Université de Caen Normandie, France

jacques.beziat@unicaen.fr

RÉSUMÉ

Les appels à projets « campus numériques français » au début des années 2000 soulignent une volonté politique d'impulser le développement de la formation à distance en enseignement supérieur. Après un bref retour historique sur les faits marquants de cette époque, les auteurs reviennent sur le destin singulier du premier campus numérique francophone en Sciences de l'éducation FORSE (Formations et ressources en Sciences de l'éducation), qui fêtera ses vingt ans en 2021. Cette vision rétroactive conduit à retenir tout d'abord l'idée d'un passage de la notion de « paysage », qui implique une approche verticale du changement en éducation et en formation, à celle « d'écosystème » du numérique, dans lequel dominent des interrelations 
fortes et constantes entre l'ensemble des membres d'un groupe projet et le politique. À partir de ce cadre d'analyse, les auteurs explorent divers aspects de l'intégration de la formation à distance jusqu'à l'assimilation dans les pratiques ordinaires universitaires : le rapport entre l'humain et les objets techniques; les relations asymétriques et les intérêts partagés entre acteurs à des postes complémentaires qui favorisent une collaboration fructueuse; enfin le développement des activités technopédagogiques qui se jouent également dans l'expérimentation et la recherche-action. Ainsi, l'importance de la prise en compte du réel de l'activité et de ses dimensions contextuelles comme conditions essentielles des rapports stabilisés entre les pratiques humaines et les objets techniques.

Mots-clés : campus numérique FORSE, formation à distance, histoire, activités technopédagogiques, approche sociotechnique, écosystème

ABSTRACT

The "digital campuses" call for projects in the early 2000s in France show a political will to boost the development of distance learning in higher education. After a brief historical review of the highlights of this period, the authors return to the unique fate of the first French-speaking digital campus in Educational Sciences, FORSE (Formations et ressources en Sciences de l'éducation / Education Science Training and Ressources), which will celebrate its twentieth anniversary in 2021. This retroactive vision allows us first to retain the idea of a shift from the notion of "landscape" which implies a vertical approach to change in education and training, to that of the digital "ecosystem", in which solid and constant interrelationships dominate between all the members of a project and politics. From this analytical framework, the authors explore various aspects of the integration of distance learning until their assimilation into regular university practices: the relationship between human and objects; asymmetric relationships and shared interests between actors in complementary positions that promote fruitful collaboration; finally, the development of educational technology activities used in experimentation and action research. Thus, the importance of the reality of an activity and its contextual dimensions are essential components to consider to stabilize relations between human practices and technical objects.

Keywords: FORSE digital campus, distance learning, history, educational-technology activities, socio-technical approach, ecosystem

\section{RESUMEN}

Las convocatorias de proyectos del "campus digital francés" de principios de la década de 2000 muestran una voluntad política de impulsar el desarrollo de la formación a distancia en la educación superior. Tras un breve repaso histórico de los momentos más destacados de este período, los autores consideran el destino singular del primer campus digital francófono de Ciencias de la Educación, FORSE (Formations et ressources en Sciences de l'éducation / Formación y Recursos en Ciencias de la Educación), que cumplirá su vigésimo aniversario en 2021. Esta visión retroactiva permite tratar, en primer lugar, el cambio de la noción de "paisaje" (enfoque vertical del cambio en la educación y la formación) a la de "ecosistema" digital, en el que existen interrelaciones fuertes y constantes entre todos los integrantes de un proyecto y grupo político. A partir de este marco analítico, los autores exploran diversos aspectos de la 
integración de la formación a distancia hasta su asimilación en las prácticas universitarias habituales: la relación entre objetos humanos y técnicos; las relaciones asimétricas y los intereses compartidos entre actores en posiciones complementarias que promueven una fructífera colaboración; y, finalmente, el desarrollo de actividades tecno-educativas a partir de la experimentación y de la investigación-acción. Se pone de relieve, pues, la importancia de tener en cuenta la realidad de la actividad y sus dimensiones contextuales, como condiciones esenciales para las relaciones estabilizadas entre las prácticas humanas y los objetos técnicos.

Palabras clave: campus digital FORSE, formación a distancia, historia, actividades tecnoeducativas, enfoque sociotécnico, ecosistema

\section{Introduction}

Cet article s'intéresse à l'un des campus numériques français encore existant après près de vingt ans d'activités, soit le campus numérique FORSE (Formations et ressources en Sciences de l'éducation), né de l'appel à projets de 2001 et impulsé par Hélène Godinet et Jacques Wallet'. Pour participer au développement de ce campus depuis plusieurs années et qui a donné lieu à de nombreuses publications (Verquin Savarieau et Daguet, 2020, 2012; Albero et Simonian, 2019; Verquin Savarieau et al., 2019; Simonian et Verquin Savarieau, 2016; Béziat, 2012; Deceuninck, 2007; Thibault, 2007; Wallet, 2007; Averous et Touzot, 2002), nous pouvons faire le constat d'une nécessaire articulation entre les acteurs de la formation et leurs représentants institutionnels, tous deux s'articulant et négociant pour développer les conditions permettant à ce campus d'être pérenne. Analyser ces conditions implique de se détacher des pratiques technopédagogiques pour proposer un cadre d'analyse écologique où les propriétés constitutives de cet environnement se transforment au fil du temps. L'écologie est comprise dans son acception première comme le rapport entre les individus d'une espèce (ici les enseignants-chercheurs), l'activité organisée de cette espèce (ici la formation à distance) et l'environnement dans lequel se produit cette activité (ici l'université). Dans son acception contemporaine, l'écologie considère dans son rapport à l'activité humaine de travail ou d'apprentissage l'environnement comme le produit et la condition de la réalisation. Positionner le campus numérique FORSE dans une telle acception revient à considérer l'environnement universitaire (des injonctions politiques à la culture des acteurs et à leur organisation), comme des espaces-temps où se produit un certain nombre de transformations des propriétés de ce même environnement à partir des acteurs qui s'y développent. Ce n'est pas l'adaptation dynamique d'un être vivant à son milieu dont il est prioritairement question, mais du lien mutuel et indissociable entre les acteurs de cet environnement, leur activité et les caractéristiques de leur contexte.

Plus spécifiquement, il s'agit d'analyser les propriétés structurantes qui constituent ce lien et celles qui se modifient du fait d'entités qui interagissent : la modification d'une des propriétés relationnelles se répercutant, à terme, sur l'environnement tout entier. Une double compréhension est dès lors fondamentale : celle des propriétés qui constituent l'environnement technique et humain du campus numérique FORSE et les propriétés qui produisent cet ensemble relationnel au sein de l'environnement FORSE, mais aussi de l'université (un environnement socioculturel régi par des lois, une culture, des

\footnotetext{
${ }^{1}$ L'histoire que nous relatons dans cet article n'aurait pu voir le jour sans la persévérance et la volonté indéfectibles ainsi que la précieuse collaboration de nombreux enseignants-chercheurs, dont celle de Jean Houssaye et de Thierry Ardouin, mais aussi, pour l'Université de Lyon 2, celle de Michel Develay et de Jean-Claude Régnier. Qu'ils en soient ici remerciés.
} 
règles formelles et informelles, un mode d'organisation). La théorie écologique ici proposée ne peut donc être réduite à une théorie des relations, puisqu'elle nécessite d'étudier la dynamique historicosocioculturelle et historico-sociotechnique des acteurs et des institutions (Albero et Brassac, 2013). De ce fait, le numérique, comme tout artefact, s'inscrit dans un ensemble fonctionnel sociotechnique et socioculturel à partir de processus progressifs d'acculturation sociotechnique et socioculturelle tant en ce qui concerne les acteurs que les institutions (Linard, 2003). C'est bien dans cette orientation que s'inscrit le présent propos, qui se centre sur l'histoire d'un groupe dans ses manières de se coordonner et de s'organiser, mais surtout de créer un sens commun tout en trouvant des manières efficientes de réaliser une activité. Cette histoire permet d'identifier des propriétés structurelles interdépendantes de l'environnement de ces acteurs, ici l'université, qui ont été à la source de controverses et de développement de ce campus numérique, que cela concerne les dimensions culturelles, organisationnelles ou productives : démocratisation de l'accès aux savoirs, marchandisation du savoir, modèle économique et rentabilité des formations, reconnaissance de l'enseignement à distance dans le statut de l'enseignant-chercheur, etc.

C'est pourquoi la centration sur l'aspect technique que sous-tend le terme « campus numérique » ne peut laisser penser que les seules dimensions de l'ingénierie technopédagogique sont centrales, notamment pour développer un environnement numérique. Or tout environnement numérique s'inscrit dans un environnement plus large où sont présents des acteurs (enseignants-chercheurs, ingénieurs pédagogiques, informaticien, étudiants) dotés de cultures comprises dans des organisations spécifiques, explicitant - pour partie - les différentes manières de comprendre le rapport à un environnement numérique et la pérennité de ce type d'environnement. Les approches sociotechniques, fort répandues en Sciences de l'éducation et de la formation, seront articulées aux approches écologiques pour mieux comprendre l'inscription historico-sociotechnique du numérique du point de vue du développement de l'activité humaine. De ce point de vue, l'environnement numérique est compris comme un médiateur de l'activité humaine qu'il incorpore. C'est pourquoi, nous appuyant sur un certain nombre de travaux portant sur les expérimentations technopédagogiques liées à ce campus, nous reviendrons sur la place centrale occupée par les relations entre acteurs dans la conception et la réalisation d'un environnement numérique d'apprentissage.

Après un bref rappel historique des faits marquants de l'appel à projets " campus numériques français ", nous retiendrons tout d'abord l'idée d'un passage de la notion d'environnement instrumenté pour l'apprentissage humain, qui implique bien souvent un tropisme du côté de la technique, à celle " d'écosystème " du numérique, dans lequel dominent des interrelations fortes et constantes entre l'ensemble de ses membres, plus nombreux, plus diversifiés dans leurs formes et leurs spécialités et surtout à une analogie au domaine de l'écologie et de l'environnement (Albero et Simonian, 2019; Simonian, 2014). On conçoit que cette approche est de nature systémique, car le paradigme écologique s'intéresse à la fois à ce qui constitue la complexité de toute personne humaine (culture, croyances, valeurs, normes, manières de vivre, etc.) et à ses interrelations avec son environnement. On considère alors que cette dernière construit son environnement qui, par voie de retour, influe sur la construction de cette personne elle-même. Ce que l'on appelle le développement résulte alors des interactions continuelles et réciproques entre l'organisme et son environnement. Ceux-ci s'influencent mutuellement et constamment, chacun s'adaptant en réponse aux changements de l'autre.

De cette manière, plus généralement, comprendre le succès ou l'échec d'un " environnement numérique » renvoie à ce qui constitue le fondement des interrelations entre des acteurs qui le font vivre et, dans le cas présenté, à des établissements différents. Cela permet également d'interroger les transformations des rapports entre l'humain et les objets techniques, et de mieux comprendre les controverses sur le plan idéologique et organisationnel, dans un contexte où les questions de pédagogie universitaire soulignent d'importantes évolutions, puisqu'il s'agit avant tout d'un consortium construit autour de deux universités partenaires (Bédard et Béchard, 2009; Béziat, 2012; Lameul et Loisy, 2012; Lison et Jutras, 2014; Annoot, 
2014; Verquin Savarieau et Daguet, 2020). II semble donc important de formaliser des déterminants écologiques qui permettent à un environnement formatif de devenir pérenne, où le dialogue entre l'acteur de terrain et le politique est aussi fondamental que l'inscription socioculturelle d'un objet technique dans l'histoire des institutions et des activités.

\section{De la lente démocratisation d'Internet au début des années 1990 aux appels à projets « campus numériques français ", l'enseignement supérieur en mutation}

L'arrivée d'Internet fut, pour l'enseignement supérieur, un moment déterminant pour un questionnement autour des évolutions à conduire des pratiques d'enseignement universitaire existantes. À la demande du ministre de l'Enseignement supérieur et de la Recherche, une mission sur l'enseignement à distance et les " nouvelles technologies », comme on disait à l'époque, est confiée à Maryse Quéré, professeure des universités en informatique. Cet important travail donnera lieu en juin 1994 à la publication du « rapport Quéré » (1994), dans lequel il est préconisé tout d'abord une orientation de l'enseignement supérieur vers un "sur mesure ", sans pour autant encore imaginer réellement le développement d'une offre d'enseignement à distance.

\section{Le rapport Quéré : Vers un enseignement supérieur français sur mesure, ou le temps de l'utopie technologique}

Le vaste chantier qui s'ouvrait alors, et qui n'est toujours pas achevé à ce jour, visait à apporter des réponses adaptées au début de la massification des effectifs des étudiants, notamment en premier cycle universitaire, afin de remédier aux forts taux d'abandons et d'échecs constatés. Parmi les propositions présentées, nous retiendrons tout particulièrement le souhait de l'essor des usages des technologies de l'information et de la communication dans les pratiques d'enseignement, afin de favoriser la diversification des modes d'accès au savoir. Pour ce faire "l'enseignement sur mesure " devait reposer sur le développement de ressources pédagogiques médiatisées et produites de façon industrielle pour satisfaire le plus grand nombre, tout en diminuant les coûts de l'enseignement supérieur (Glikman, 2002; Grevet, 2005). Une des conséquences était de transformer l'université dans ses formes d'organisation sociale et de productions (Moeglin, 2016; Jacquinot et Fichez, 2008), incluant les cultures affiliées tant en ce qui concerne les acteurs que les établissements universitaires. D'ailleurs, Quéré soutient la nécessité de produire de la " connaissance médiatisée » (Quéré, 1994, p. 3) avec l'idée de la création d'un service d'enseignement ajusté à tous les publics, que ce soit celui de la formation initiale ou continue, de la formation en présence ou à distance, jusqu'à privilégier des temps d'apprentissage en autoformation afin de favoriser une plus grande accessibilité des universités. Pour cela, quatre grands principes d'action furent retenus (Quéré, 1994, p.10) :
1) Production de ressources d'autoformation (tous médias);
2) Création dans les sites universitaires d'un maillage de centres de ressources;
3) Télédistribution interactive d'activités (parties de cours mises à distance);
4) Télédiffusion de cours (par la radio, la télévision, le numérique).

Nous retiendrons de ce rapport la surdétermination technologique comme principal vecteur de transformation de l'environnement, confusion encore fréquente dans bien des discours actuels. En Sciences de l'éducation et de la formation, si la croyance dans l'efficacité de certains processus 
d'autoformation ou d'interformation ne fait pas débat, il n'y a pas de naïveté non plus. En un mot : la mythique appropriation idéale des connaissances par l'apprenant n'est pas liée au seul choix, fût-il judicieux, d'un logiciel support (Jacquinot, 1993; Linard, 1990).

Chaque nouvelle technologie alimente une utopie : l'outil de référence est associé au rêve d'une certaine école ou d'une certaine société [...] comme toujours, les développements technologiques loin de remplacer l'enseignant [...] ne font qu'exiger de lui plus de maîtrise dans la connaissance des processus d'apprentissage et toujours plus d'imagination, [...]

(Jacquinot, 1985, p. 17)

Plus largement, la surdétermination permanente de la logique instrumentale, au détriment de celle de l'usager, est à remettre en question, notamment lorsqu'un instrument est considéré comme ce qui va modifier un environnement. En effet, si besoin en était, rappelons que le processus de genèse instrumentale tient compte des dimensions environnementales et, plus spécifiquement écologiques, en considérant que tous les acteurs d'un même environnement peuvent transformer matériellement et/ou conceptuellement une propriété de l'artefact en fonction de conceptions. S'il est possible de considérer que toute action sur un instrument agit sur l'environnement, mais aussi sur l'utilisateur dans sa manière de comprendre l'environnement dans lequel il agit, vit et évolue, ce type d'analyse implique de comprendre de quelles manières, et pour quelles raisons, l'institution s'empare des modifications qui sont susceptibles de se produire en concertation, plus ou moins effective et injonctive, avec les acteurs concernés (évolution ou tolérance par rapport à la reconnaissance de l'enseignement à distance dans le service statutaire de l'enseignant par exemple, ou encore en termes de politique financière pour la formation à distance). En d'autres termes, la logique instrumentale s'inscrit dans une sociologie des usages (Linard, 2001) nécessairement située (Akrich, Callon, Latour, 2006) où se jouent des processus de signification et de compréhension qu'ont les acteurs de leur devenir et de celui de leur propre environnement de travail.

En témoigne la proposition radicale du rapport Quéré, 1994, p. 10) :

Un usage plus radical peut être envisagé, qui supprime complètement l'enseignant. La connaissance est disponible au travers de réseaux de médiathèques. Le principal travail de l'étudiant est la recherche des informations, leur acquisition, et une activité de synthèse qui se traduit par une production (devoir, exposé, projet). L'enseignant se borne alors à son rôle de vérificateur.

Pour autant, malgré cette conception utopique d'une technologie qui remplacerait totalement l'enseignant, le campus numérique FORSE - ici étudié - s'est développé au fil des années par la stabilisation, durant une certaine période (Albero et Simonian, 2019), de normes intermédiaires conduisant les acteurs à négocier - au sein du consortium, mais aussi au sein des acteurs de chacune des institutions partenaires - ce qui est central dans leur métier, sous couvert de l'environnement numérique. Ce sont les résultats de ces controverses (Callon, 1986; Latour, 1992) qui fournissent les propriétés modifiées de leur environnement, mais aussi celles, inaliénables, incorporées dans l'histoire de la profession.

\section{Les appels à projets " Campus numériques français " au début des années 2000 , ou le temps des transformations profondes du paysage universitaire}

Du rapport Quéré (1994) aux trois appels à projets « campus numériques » de 2000, 2001, 2002, marqués par la volonté politique de l'intégration des TIC en éducation et en formation, a succédé une volonté « de développement intensif de la formation ouverte et à distance (FOAD) » (Averous et Touzot, 2002, p. 8). En France, comme dans de nombreux pays européens, l'impulsion est donnée afin de soutenir l'usage 
des TIC dans les établissements d'enseignement supérieur et de favoriser l'émergence d'une offre de formation à distance. II est vrai que depuis la création de 22 centres de télé-enseignement universitaire dans les années 70 (Thibault, 2002), c'était la première fois que le ministère incitait à développer massivement la formation à distance au cœur de ces établissements. II s'agissait donc de moderniser le service public d'enseignement supérieur, en créant et en promouvant une offre française compétitive sur le marché international, inscrite dans une logique cohérente de regroupements interétablissements portée par des consortiums, ce que privilégie depuis toujours le modèle du développement de la formation à distance à la française. À noter qu'il n'a jamais été question en France d'opter pour la création d'établissements universitaires totalement à distance, mais que les formations universitaires à distance sont dispensées dans des universités traditionnelles, comme l'écrit Mathilde Miguet (2011). Pourtant dans certains pays européens, des universités unimodales en formation à distance voient le jour à cette époque; c'est le cas de l'Allemagne avec la FernUniversität in Hagen², du Royaume-Uni avec l'Open University³, de l'Espagne avec l'Université nationale en éducation à distance ${ }^{4}$ ou l'Université ouverte de Catalogne $(\mathrm{UOC})^{5}$, ou bien encore du Portugal avec l'Université Alberta 6 , etc.

II s'agissait par conséquent de s'aligner sur l'évolution en cours de la société, dans laquelle l'intégration des TIC progressait dans les usages sociaux. Par analogie avec un campus physique, les campus numériques devaient rassembler des moyens humains et techniques importants afin de permettre d'offrir et de diversifier les services offerts aux étudiants, présents ou non physiquement dans un établissement d'enseignement supérieur. Ces services, à construire dans une grande variété, pouvaient aller de la simple diffusion de supports pédagogiques sous forme numérique à la formation ouverte assurée plus ou moins à distance (FOAD), en passant par le tutorat et le travail de groupe à distance, et par des services administratifs ou culturels variés. II s'agissait donc de mettre à jour les moyens et méthodes d'enseignement en exploitant notamment les ressources offertes par les technologies de l'information et de la communication. L'idée étant de « permettre d'améliorer l'enseignement en l'enrichissant d'une réelle ingénierie de formation reposant notamment sur la modularisation des cursus, la diversification des pratiques d'accompagnement des étudiants comme sur la flexibilité des parcours » (Thibault, 2001, p. 5a).

Ce projet de développement de l'enseignement à distance à l'université a suscité de nombreux débats contradictoires autour de la pertinence de cette démarche. Parmi les critiques fréquemment exprimées, nous soulignons une suspicion de base sur des principes économiques qui pourraient faire de la formation à distance un levier pour la marchandisation de l'enseignement supérieur (Thibault, 2001; Aubert, 2001)', mais également le risque qu'elle comporte un taux élevé d'abandons ou d'échecs, tant pour des étudiants que des enseignants mal préparés, ou bien encore une dépersonnalisation de l'enseignement. Ces trois éléments remettaient en question l'héritage socioculturel de l'activité des enseignants-chercheurs en

\footnotetext{
2 Découvrir cette université : https://www.fernuni-hagen.de/

${ }^{3}$ Découvrir cette université : Distance Learning Courses and Adult Education - The Open University

${ }^{4}$ Découvrir l'UNED : UNED | Universidad Nacional de Educación a Distancia - Enseñanza Online

${ }^{5}$ Découvrir l'UOC : UOC (Universitat Oberta de Catalunya)

${ }^{6}$ Découvrir cette université : Universidade Aberta (uab.pt)

${ }^{7}$ Un des enjeux est de développer un nouveau modèle économique global de l'enseignement supérieur auquel pense le Ministère (Thibault, 2001, p.5) « qui repose sur des investissements pédagogiques importants intégrant l'existence de publics différents qui accèdent à l'université selon des modes divers (faibles coûts pour les étudiants en formation initiale, coûts adaptés aux autres) ", afin de ne pas réserver l'accès au secteur marchand de la formation aux entreprises privées spécialisées uniquement. Dans ce contexte, le CNED, qui a signé un protocole d'accord avec la Conférence des présidents d'université (CPU), la Conférence des directeurs d'écoles et formations d'ingénieures (CDEFI), la Conférence des directeurs d'instituts universitaires de formation des maîtres (CDIUFM), devient un partenaire incontournable de nombreux projets de campus numériques, afin que «le service public d'éducation devienne l'un des acteurs majeurs de ce marché hautement concurrentiel » (Aubert, 2001, p. 3). En tant qu'acteur incontournable de l'enseignement par correspondance à la suite de sa création en 1939 dans le contexte de l'occupation et de la nécessité de scolariser de nombreux enfants réfugiés, ce service de cours par correspondance, imaginé comme temporaire, correspond à une vraie demande sociale même après la guerre et il continuera à se développer jusqu'à jouer un rôle moteur dans le développement de la formation à distance en France jusqu'à aujourd'hui.
} 
France basé sur la démocratisation de l'accès aux savoirs (et non à sa marchandisation), la réussite du plus grand nombre (et non un élitisme) et la personnalisation des savoirs (et non un processus d'industrialisation à parcours unique). Toujours est-il que ces campus numériques font craindre des " moulinettes à diplômes virtuels " (Thibault, 2002); Dridi et Chouinard, 2003) ou encore des " usines à formation » (Lapalme, 2003), tout en remettant en question la reconnaissance officielle de l'activité de l'enseignement à distance dans le statut des enseignants-chercheurs, notamment pour que de nouvelles modalités de travail puissent être stabilisées (Paivandi, 2009). II faudra attendre $2009^{8}$ pour qu'un premier référentiel comporte une première réflexion sur les équivalences horaires pour l'ensemble des activités réalisées par les enseignants-chercheurs français. C'est à présent aux universités devenues autonomes qu'il revient d'établir un cadre régissant ces équivalences, ou alors de tolérer cette activité sans cadre officiel au niveau de l'université mais au niveau du consortium, pour les activités réalisées à distance dans toute leur diversité.

À cette même époque du début des années 2000, c'est aussi la formation professionnelle qui connaît une véritable révolution en intégrant la possibilité d'une "validation des acquis de l'expérience " dans les cursus d'enseignement. II ne s'agit plus uniquement d'une demande sociale de qualification, mais de la recherche d'une reconnaissance de l'expérience qui ouvre à une promotion sociale et à une égalité des chances selon la Loi. Ainsi, la VAE intègre la formation tout au long de la vie et s'inscrit, pour certains, dans une logique « de démocratisation des études " (Gauttier, 2012, p. 1110), et pour d'autres comme un moyen de « resserrer les liens entre le monde universitaire et les entreprises et de renouveler les pratiques pédagogiques " (Pinte, 2008, p. 150) ou comme " un espace de travail commun fait de dialogue et de négociation entre deux mondes que l'on estime a priori hétérogènes l'un à l'autre " (Mayen et Métral, 2008, p. 188). Cette VAE, en devenant la quatrième voie de certification à côté de la formation initiale et de l'apprentissage, devient également un levier potentiel de la professionnalisation de l'offre de formation de l'enseignement supérieur (Verquin Savarieau, 2013; Wittorski, 2012), non sans susciter de profondes interrogations sur les liens que l'on peut faire entre référentiels de formation, exploitation et valorisation de ces derniers.

Outre les lois portant sur la validation des acquis, l'idée d'une éducation et d'une formation tout au long de la vie (EFTLV) introduite par la Commission européenne à peu près à la même période (2001) désigne «toute activité d'apprentissage entreprise à tout moment de la vie, dans le but d'améliorer les connaissances, les qualifications et les compétences, dans une perspective personnelle, civique, sociale et/ou liée à l'emploi ». Ainsi, la Commission européenne (2001) met l'accent sur l'apprentissage aussi bien dans sa durée que dans ses formes : "L'apprentissage qui va de l'enseignement préscolaire jusqu'à l'après retraite ("du berceau au tombeau"), et ouvre toute forme d'éducation (formelle, informelle ou non formelle) ».

La formation à distance peut alors être ici comprise comme une modalité pertinente malgré des conceptions a priori opposées : personnalisation pour la formation continue versus standardisation pour la formation à distance, développement des compétences versus transmission des savoirs, etc. Mais, peu importe, les incitations au développement de la formation à distance à partir de ces appels d'offres " campus numériques » ont généré des consortiums dont les finalités sont bien différentes selon que cela concerne les campus Pegasus, Canege, FORSE, etc. Dans leur rapport au ministre de l'Éducation nationale, Averous et Touzot (2002, p. 13) indiquent qu'à cette époque, « les effectifs d'apprenants inscrits à des dispositifs de FOAD en France et relevant du ministère de l'Éducation nationale sont en lente progression : 165000 dont 87000 en préparation à des concours de la fonction publique pour le CNED et les établissements d'enseignement supérieur, auquel il faut ajouter environ 7000 pour le CNAM ». Mais

\footnotetext{
${ }^{8}$ Référentiel national d'équivalences horaires applicable aux enseignants-chercheurs français : Légifrance, Publications officielles, Journal officiel, JORF, $n^{\circ} 0187 \mathrm{du}$ 14/08/2009 (legifrance.gouv.fr)
} 
nous retiendrons surtout que cette offre française de formation à distance dans l'enseignement supérieur repose encore à l'époque principalement sur l'utilisation de la voie postale même si celle-ci est associée progressivement à d'autres supports de communication (téléphone, Minitel, audiovisuel, vidéogrammes et Internet), comme c'est le cas du campus FORSE au début des années 2000. Toutefois, très rapidement, les campus, dans une logique d'industrialisation éducative (Mœglin, 2016; Mœglin et Thibault, 2009), devront remettre en question les choix de plateformes, et la formation à distance peut alors accompagner progressivement les logiques de massification des effectifs universitaires. Pour le campus FORSE, qui répond à une vraie demande sociale, les étudiants seront au rendez-vous dès le début avec, de 2001 à 2004 , les effectifs suivants : $862,1010,938$ et $1000^{\circ}$, ce qui constituait, pour l'époque, un véritable succès.

\section{Retour historique synthétique sur le premier campus numérique francophone consacré aux enseignements en Sciences de l'éducation : FORSE (Formations et ressources en Sciences de l'éducation)}

L'histoire du campus FORSE s'inscrit dans le prolongement de l'offre de formation du présentiel des deux universités partenaires, basé sur une double volonté : favoriser la démocratisation de l'accès aux savoirs et l'éducation tout au long de la vie pour des publics ne pouvant accéder à la formation en présentiel. Les trois partenaires de FORSE (Université de Rouen, Lyon 2 et le CNED) ${ }^{10}$, partageant ces valeurs, s'associent autour d'un projet ambitieux : celui de créer le premier campus francophone entièrement consacré aux Sciences de l'éducation. Pour ce faire, l'Université de Rouen, en partenariat avec le CNED, initie dès 1997 la mise à distance du DESS Ingénierie de la formation, qui existait jusqu'alors en modalité présentielle (Wallet, 2007; Miguet, 2011). Puis trois ans plus tard, ce partenariat s'élargit avec l'arrivée de I'Université de Lyon 2 et l'ouverture d'une licence de Sciences de l'éducation à distance. Ainsi, si l'offre de formation qui intégrera le campus numérique FORSE préexiste pour partie à l'appel à projets de 2001, il est notable que les encouragements apportés par le ministère de l'Éducation et de la Recherche afin de développer une offre nationale, et le budget alloué pour trois ans, a su persuader les partenaires de se lancer dans l'aventure (Thibault, 2007, Wallet, 2007).

Cette mise de fonds a permis la médiatisation des contenus (coûts de développement des ressources numériques) sur la plateforme de formation (WebCT d'abord, puis Moodle depuis 2019) prise en charge par le CNED, puisqu'à l'époque les universités n'étaient prêtes ni à gérer une plateforme numérique, ni à médiatiser les corpus de savoirs, ni à rémunérer le tutorat. Ce n'est qu'en 2011 qu'une partie du tutorat a pu être prise en charge par l'université, s'inscrivant dans le changement de statut de 2009 reconnaissant officiellement l'enseignement à distance dans le statut des enseignants-chercheurs, et dans l'acceptation des universités de valoriser le tutorat comme une forme d'enseignement spécifique à la distance, notamment pour promouvoir la réussite des étudiants tout en valorisant son coût chargé. Mais revenons en 2001, date où le campus numérique FORSE voit le jour (figure 1) dans un consortium associant les universités de Rouen, Lyon 2 et du CNED. Les formations proposées à distance et en présentiel ont des contenus identiques. Un an plus tard, ce sera l'ouverture de la maîtrise en Sciences de l'éducation (futur master 1) (2002). Et c'est sans aucun doute l'année 2004 qui marquera le plein développement de ce partenariat, en réussissant non seulement le passage de l'ensemble de l'offre au « Lmd », pour « licence, master, doctorat ", mais également la création d'un deuxième master cette fois-ci de recherche, le MARDIF (Master de recherche international francophone, devenu le MIFEF (Master international francophone en éducation et formation) en 2011, depuis que les masters sont devenus « indifférenciés ",

\footnotetext{
${ }^{9}$ Sources : comités de pilotage.

10 Vidéo de présentation de FORSE, témoignant de la participation de nombreux enseignants-chercheurs reconnus dans la discipline (JeanPierre Astolfi, Thierry Ardouin, Jean Houssaye, Jacques Wallet, etc.) : Présentation du Campus numérique FORSE, YouTube, consultée en ligne le 6 décembre 2020.
} 
soit ni professionnels ni de recherche), élargissant le recrutement de l'équipe des enseignants à la francophonie (Belgique, Canada, Suisse et France) et à 13 universités (Houssaye, 2007, p. 107).

\section{Figure 1}

Élargissement et transformations de l'offre de formation au fur et à mesure de l'élargissement du consortium

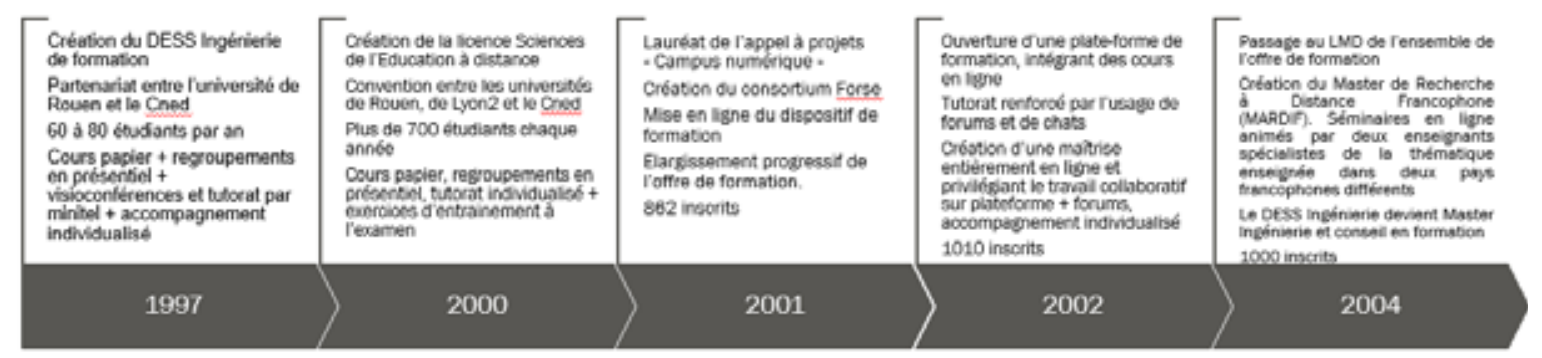

Le dispositif de formation mis en œuvre est propre à chaque diplôme et repose sur les éléments suivants : ressources pédagogiques, ressources méthodologiques, exercices d'entraînement à l'examen et leur corrigé, scénarios pédagogiques, accompagnement tutoré, regroupements présentiels (comportant, selon le cas, des compléments de cours et/ou des travaux dirigés et/ou des examens). La constitution de cette offre de formation conduira à observer une inversion du profil des étudiants inscrits dans le département des Sciences de l'éducation; la part des effectifs « présentiels » décroit, tandis que celle des « distanciels » représente progressivement la majorité des étudiants. S'il est vrai que la formation à distance élargit l'espace de recrutement des universités, c'est aussi l'opportunité de la diversification des publics qui l'accompagnent qui favorise une augmentation des effectifs globaux constatés. Car le développement de l'offre à distance s'accompagne également du développement des effectifs de la formation professionnelle continue, déjà engagés dans la vie active et qui cherchent à réaliser des études tout en continuant de travailler.

La modalité dite "à distance " apporte donc une réponse à leurs besoins spécifiques, notamment quand on sait que « ce qui compte dans les formations à distance, c'est la présence! » Cette citation de Thierry Ardouin (2007, p. 82) fait écho au fait qu'au sein des formations du campus FORSE, une logique d'hybridation (à présent très en vogue) existe dès son origine, soit en intégrant des regroupements présentiels qui ont pour mission d'accompagner les étudiants dans leurs apprentissages et de les guider ainsi vers la réussite et l'acquisition de leur diplôme. Ils répondent également à une logique de professionnalisation de l'offre de formation universitaire, en favorisant une socialisation et la construction d'un réseau de pairs susceptible de devenir un réseau professionnel à l'issue de leur formation. Par conséquent, les enquêtes réalisées auprès de ces publics montrent que les inscrits à distance n'auraient pu suivre des études en présentiel, ce qui aura le mérite de rassurer certains membres des équipes universitaires, dont la peur était de vider les amphithéâtres.

Enfin, poursuivant sa politique de partenariat et d'ouverture à une nouvelle échelle géographique, le campus FORSE signe, en août 2004, un partenariat avec l'Agence Universitaire de la Francophonie (AUF), ce qui permet l'octroi d'une trentaine d'allocations par an sur l'ensemble des formations, mais principalement en master 1 et 2 pour des étudiants de pays francophones (Porot-Viaouet, 2007), avec des sites de regroupements fréquemment organisés, selon les années, au Burkina Faso et au Cameroun. Audelà de la distance géographique ou de la fracture numérique, cette ouverture du campus aux pays dits " du Sud » souligne les difficiles conditions d'accès au réseau Internet ou à l'électricité dans cette zone géographique; c'est pourquoi les étudiants qui peuvent rejoindre un site de regroupement bénéficient du soutien logistique et de l'appui des ressources documentaires ou numériques des campus numériques 
francophones mis en place par l'AUF. Si le référent de site a toujours été recruté parmi les anciens étudiants du campus FORSE, il est notable qu'un enseignant-chercheur pût une fois l'an rejoindre le site de regroupement pour le coanimer, manifestant de cette manière toute l'attention accordée à chaque étudiant du campus. Plus encore, l'un des référents de site a été nommé ministre de l'Éducation nationale de son pays, preuve s'il en est que la distance en formation peut être à l'origine de parcours d'exception. La cohérence du discours institutionnel était également portée par une visioconférence qui était organisée lors de chaque regroupement, permettant ainsi à chaque site de regroupements (maximum au nombre de cinq) d'échanger et de se fédérer au-delà des frontières.

De cette manière, le rapport de l'ERTe « Modèles économiques et enjeux organisationnels des campus numériques" (Deceuninck, 2005; 2007) souligne que le campus FORSE constitue une "réussite durable "; que devrions-nous dire plus de quinze années plus tard? La diversité des destins des campus numériques amène ce même auteur (Deceuninck, 2007, p. 6) à s'interroger sur la culture du monde universitaire et les transformations que les campus pourraient générer, même si, pour lui, l'analyse des campus demanderait à être recadrée dans un temps plus long. II précise que « les acteurs qui répondent à un appel d'offres s'engagent plus sur un principe d'action que sur une visée de résultats; plus intéressés par l'innovation et le développement de prototypes que par la généralisation de dispositifs, ils sont plus proches du monde de l'expérimentation et de la recherche que de celui de l'ingénierie et de l'administration ». Sur cet aspect, il a pu se tromper, cependant il a vu juste lors qu'il affirme que « les dispositifs de formation qui ont vu le jour relèvent en effet du développement dans le monde universitaire d'une nouvelle culture de pilotage par projets, lesquels sont limités dans le temps, avec désengagement annoncé de l'État. Un tel changement ne relève pas d'une temporalité courte ". C'est sur ce point des transformations générées par la vie du campus numérique FORSE au sein des institutions partenaires que nous voudrions développer à présent notre propos.

\section{Développement de la formation à distance dans l'environnement universitaire : entre innovation et ingénierie, nécessité de repenser les rapports entre sujet - objets techniques et environnement}

Nous désirons mettre en évidence la manière dont se sont établies les relations entre acteurs au sein de ce campus FORSE afin de souligner les propriétés transformatives de la formation à distance sur l'environnement universitaire. Si nous avons cherché à mettre en avant précédemment certaines de ces propriétés économiques et structurelles, ici ce sont les conceptions et les pratiques pédagogiques universitaires dont il est question, interdépendantes des propriétés susmentionnées, notamment en termes de scénarisation et de tutorat, impliquant le passage d'une logique d'instruction à celle de l'apprentissage où, rappelons-le, la transmission des connaissances universitaires s'est historiquement construite avec un enseignement magistral (Denis, 2003; Barbot et Combès, 2006; Kim et Verrier, 2009; Baron, 2010). Pour ce faire, nous reviendrons également sur quelques travaux de recherche soulignant les grandes orientations des recherches en technologies de l'éducation effectuées autour du campus FORSE (Develay et Godinet, 2007; Eneau et al., 2008; Béziat, 2012). 


\section{Le rapport entre l'humain et les objets techniques, un débat ancien toujours d'actualité}

L'histoire tout autant que l'actualité donnent raison aux travaux qui ont, de longue date, montré l'ambivalence du rapport de l'humain aux objets techniques puis, aujourd'hui, au numérique. Toute avancée humaine se paie de déqualifications et les changements occasionnés produisent parfois autant de déstructurations ou de destructions qu'ils ne causent de bienfaits ou de progrès. Ce qui est vrai dans bien des domaines d'activités (écologie, santé, industrie, commerce, etc.) l'est aussi en matière d'éducation et de formation. Parmi les conditions d'émergence des environnements numériques d'apprentissage, nous retiendrons notamment la nécessité de concevoir une ingénierie de formation que nous identifions comme étant la production de compétences et de savoirs en lien avec les situations professionnelles ou sociales. L'ingénierie n'existe pas en soi; c'est bien l'objectif de production de savoirs et de compétences qui en est la finalité première, par la mise en œuvre d'une réflexivité cherchant à déjouer l'incertitude croissante du monde économique. Si ces savoirs et compétences sont liés au contexte d'usage, qu'il soit professionnel ou social, ils ne se réduisent absolument pas à des connaissances opérationnelles et techniques, mais les intègrent dans des connaissances plus vastes et des savoirs génériques. Autrement dit, la finalisation des savoirs contextualisés n'est pas uniquement opératoire, mais plus largement sociétale, et reste tributaire de logiques financières. De cette façon, « pour tenter de rendre explicite une autre manière d'analyser les dynamiques plus complexes qui se déploient dans ces environnements de formation, il s'avère utile de mettre en évidence quelques-uns des aspects qui apparaissent cruciaux, alors même que, par habitus ou par commodité, ils sont régulièrement négligés par nombre d'acteurs. C'est le cas du dialogue majorant avec le politique » (Albero et Simonian, 2019, p. 26). De cette façon, pour le faire mentir, oui, il s'agissait bien d'innover, mais l'ingénierie de la FOAD et les conditions administratives et financières ont bien souvent été déterminantes dans les conditions de réussite du campus FORSE.

Dans ce sens, l'inscription institutionnelle des campus numériques présente à la fois des similitudes et des différences par rapport aux autres dispositifs de formation à distance, comme c'est le cas, par exemple, des centres de télé-enseignement et des services de formation continue dont le statut est clairement établi en tant que services communs qui ont la particularité de pouvoir générer des ressources financières. Dans le cas des campus, il en va autrement. Lors de leur création, certes c'étaient les présidents des universités qui en avaient officiellement la responsabilité ainsi que le recteur du CNED, mais le plus souvent, ils déléguaient très largement la conduite de ceux-ci à des chefs de projet dont les liens avec les autres composantes des établissements (Unité de Formation et de Recherche (UFR) et services communs) n'étaient pas toujours définis avec précision. Ainsi, dans le cas de FORSE, il est vrai qu'il existait une certaine zone de latitude laissée à son chef de projet au sein des universités, celle de Rouen notamment, ce qui a permis le développement de diverses initiatives, dont la création de sites de regroupements, y compris à l'étranger, et des déplacements ponctuels d'enseignants-chercheurs pour les coanimer (dont nous avons déjà parlé), mais surtout la constitution d'une équipe de contractuels dont les missions sont consacrées au campus (administrateurs plateformes et coordonnateurs pédagogiques), dont le départ à l'issue de leur contrat génère chaque fois la même question : le projet est-il durable et mérite-t-il qu'on lui accorde un recrutement? De cette manière, si la réussite est durable, même après vingt années d'existence, l'équipe projet du campus n'est toujours pas assurée de la même pérennité qu'un service commun ou qu'une UFR auxquels le campus est rattaché (UFR SHS et Service de la formation continue). Le caractère interuniversitaire et le partenariat avec le CNED dans le cadre d'un consortium, dont la convention de partenariat est renouvelée tous les cinq ans à la suite de vives renégociations, renforcent encore cette spécificité qui peut parfois le fragiliser ou tout le moins freiner son développement.

Ainsi, penser le rapport à l'objet technique comme ne relevant que de la responsabilité de l'acteur face à la machine peut être considéré comme une erreur fréquemment effectuée, car comment peut-on 
finalement désolidariser l'objet technique des facteurs culturels, sociaux et de productions? II semble que non, puisque ne pas considérer ces facteurs empêcheraient d'anticiper les mutations en œuvre ou de les reproduire, d'y préparer les institutions et les acteurs, voire de les accompagner pour qu'ils puissent contribuer eux aussi à ces évolutions dans la pleine connaissance des caractéristiques de leur activité (Engeström, 2001; Linard, 1990; Albero, Linard et Robin, 2009). Plus spécifiquement, dans le domaine du numérique éducatif, nous retiendrons que les investissements massifs sont prioritairement réalisés dans les équipements ${ }^{11}$ plutôt que dans la réflexion autour des transformations du travail que ces mutations technologiques génèrent. Cette absence de concertation peut décourager plus qu'elle ne stimule le professionnalisme des enseignants et/ou des formateurs. En ne participant ni aux processus d'achat ni à celui du renouvellement des artefacts pédagogiques en tant qu'objets médiateurs du savoir, faits à la main par ces principaux intéressés, ils peuvent se sentir disqualifiés et contraints de s'adapter à des activités pensées comme finalisées et auxquelles ils n'ont pas été associés en tant que concepteurs. Cette absence de la fonction d'anticipation de l'activité que doit comprendre toute conception d'objets les met souvent dans l'incapacité de comprendre les évolutions techniques et de pouvoir les intégrer de façon optimale. Cette situation, que l'on ne pourrait imaginer dans le domaine industriel, engendre nombre de dysfonctionnements parce qu'elle contraint l'activité à s'adapter aux objets techniques à disposition, et non l'inverse.

Ce primat de l'objet technique sur le travail réel et du budget d'équipement dans la répartition des charges ne permet pas de rémunérer le travail indispensable pour penser l'évolution de l'activité. La conséquence peut être une posture de retrait qui accompagne en réalité une perte de sens du projet pédagogique global et qui se traduit sur le plan individuel par : lassitude et découragement, désinvestissement, absence de perspective, diminution d'intérêt pour l'activité, absentéisme, maladies, douleurs chroniques, dépression, burn-out. La rationalisation extrême du travail et la transformation de l'activité pensée en mode "procédure ", à laquelle peut conduire le pouvoir accordé aux objets techniques contemporains, contraignent les professionnels à engager leur énergie et leur temps à remplir les conditions de fonctionnement requis pour pouvoir agir (demandes administratives et techniques; autorisations formelles ou symboliques; bilans et rapports; évaluations, audits et expertises, etc.). Pierre-Michel Menger (2009), qui s'est intéressé au « travail créateur », nous indique que toute forme de réussite de l'activité dépend de quatre paramètres :

1. De l'acteur lui-même (ici l'enseignant ou l'équipe pédagogique ou de projet);

2. De l'environnement de l'activité et de ses conditions de réalisation (matérielles, juridiques et politiques);

3. De la qualité du travail d'équipe qui s'affaire dans le projet échafaudé pour créer une œuvre;

4. De l'évaluation de ceux qui seront destinataires de cette œuvre (élèves, étudiants, équipe pédagogique ou de projet).

${ }^{11}$ Cette tendance avait déjà été montrée dans une enquête européenne (Albero et Thibault, 2006). 
En n'ayant plus de temps pour penser le travail créateur, sans cessation d'activité, point d'esprit libre et disponible pour penser des améliorations ou une appropriation pérenne des nouvelles pratiques. Ainsi, historiquement, toute promotion politique d'une nouvelle manière d'enseigner à partir de technologies remet en cause les logiques d'action qui visent aussi la transformation structurelle et socioculturelle d'une organisation. En définitive, nous pouvons constater que lorsque les acteurs s'en saisissent, certaines peuvent être anticipées et devenir pérennes. À l'encontre de la doxa binaire du top-down/bottom-up qui consiste à penser que la relation entre l'activité décisionnelle du politique et celle du terrain éducatif est de nature soit descendante, soit ascendante, l'analyse des processus de cocréation de réponses adaptées relève davantage d'une rencontre entre acteurs placés à des postes différents mais complémentaires (Albero et al., 2009) et qui recherchent un sens et une valorisation au projet qu'ils mettent en œuvre ensemble.

\section{Les intérêts partagés entre acteurs à des postes complémentaires favorisent une collaboration fructueuse}

Le campus FORSE fait partie des campus numériques qui ont survécu et qui se sont développés à partir d'une réelle dynamique partenariale. Pourtant, nous le comprenons, celle-ci aurait pu faire sa fragilité, d'où l'intérêt de remettre en question les éléments marquants qui conduisent à constater sa réussite. D'un projet qui ne reposait au départ que sur la bonne volonté de quelques-uns, souvent chevronnés en matière de pratiques innovantes et pouvant s'appuyer sur l'engagement de leurs établissements (Fichez, 2007), FORSE s'est intégré à la culture universitaire parce qu'il ne représentait pas de profondes transformations de prime abord. Les enseignants responsables des cours sont restés maîtres des contenus et de leurs modalités d'évaluation, notamment lors de leur réalisation dans des examens qui sont restés longtemps sur table. En revanche, c'est le CNED qui rémunère les enseignants pour les cours qu'ils ont rédigés et dont ils ont cédé les droits pour une exploitation médiatisée au départ dans des fascicules de cours papier et des devoirs à envoyer par voie postale, très rapidement pour des cours médiatisés et des copies en ligne. Nous noterons que l'héritage des cours par correspondance auquel nous pouvons associer le poids de l'écrit dans la transmission des savoirs s'amenuise avec le temps, pour être remplacé de plus en plus par des interventions synchrones réalisées en classes virtuelles. Ces cours, appelés " webinaires », ont été introduits à l'Université de Rouen Normandie dans le campus FORSE il a près de dix ans. Peu à peu, les deux universités en ont fait usage, ce qui a été très appréciable lors du premier confinement en 2020 et qui a permis de dispenser des cours en ligne pour tous les étudiants, y compris ceux du présentiel. De cette manière, comme le soulignent Gérin-Lajoie et Potvin $(2011$, p. 351) : " II existe peu de cas documentés du processus évolutif d'établissements universitaires vers la bimodalité, surtout du côté francophone ", d'où la nécessité de relater quelques faits marquants transposables à d'autres universités, afin que les acquis de certains projets puissent bénéficier au plus grand nombre. Poursuivant la lecture des travaux d'Albero et Simonian (2019), nous retiendrons tout d'abord que la gouvernance de ce campus n'est pas de la seule responsabilité du comité de pilotage et des chefs de projet (un pour les trois institutions qui le composent et une présidence tournante à l'année), mais qu'il en découle une organisation dans laquelle, chaque partenaire joue un rôle déterminant en fonction de son domaine de collaboration (tableau 1). 
Tableau 1

Domaines de collaboration établis au sein du campus numérique FORSE

\begin{tabular}{|c|c|c|}
\hline Domaines de collaboration & Activités retenues & $\begin{array}{c}\text { Apports spécifiques des } \\
\text { parties }\end{array}$ \\
\hline $\begin{array}{l}\text { Accueillir, informer et orienter } \\
\text { les prospects }\end{array}$ & $\begin{array}{l}\text { Garantir l'accueil, l'information, } \\
\text { l'orientation, la communication et } \\
\text { la gestion des apprenants par une } \\
\text { logistique appropriée }\end{array}$ & $\begin{array}{c}\text { Un site d'accueil consacré } \\
\text { exclusivement au dispositif } \\
\text { FORSE, ne privilégiant aucun des } \\
\text { partenaires }\end{array}$ \\
\hline $\begin{array}{c}\text { Assurer, à la demande des } \\
\text { parties universitaires, } \\
\text { l'hébergement et la } \\
\text { maintenance des ressources } \\
\text { pédagogiques et des espaces } \\
\text { collaboratifs }\end{array}$ & $\begin{array}{l}\text { Héberger et maintenir des } \\
\text { ressources pédagogiques et des } \\
\text { espaces collaboratifs }\end{array}$ & $\begin{array}{l}\text { Tous les partenaires, chacun en } \\
\text { fonction de ses compétences. } \\
\text { Contrat de tutorat initié par le } \\
\text { CNED lorsque l'université ne } \\
\text { pouvait pas l'assurer. Modalités } \\
\text { technopédagogiques selon les } \\
\text { choix établis par les équipes } \\
\text { universitaires. }\end{array}$ \\
\hline $\begin{array}{l}\text { Concevoir et développer les } \\
\text { dispositifs administratifs et } \\
\text { juridiques les plus appropriés } \\
\text { aux objectifs de FORSE }\end{array}$ & $\begin{array}{l}\text { Rédiger une convention de } \\
\text { partenariat qui sera mise à jour } \\
\text { tous les } 5 \text { ans. } \\
\text { Stabiliser un modèle économique } \\
\text { propre au dispositif }\end{array}$ & $\begin{array}{l}\text { Tous les partenaires, chacun en } \\
\text { fonction de ses compétences }\end{array}$ \\
\hline $\begin{array}{l}\text { Participer à la création ou à la } \\
\text { médiatisation des supports de } \\
\text { cours et autres ressources } \\
\text { nécessaires au bon } \\
\text { déroulement des formations } \\
\text { mises en œuvre dans FORSE }\end{array}$ & $\begin{array}{l}\text { Créer et médiatiser des cours } \\
\text { mettant en collaboration des } \\
\text { auteurs des deux universités } \\
\text { partenaires. Contractualiser des } \\
\text { ressources et des droits d'auteurs. }\end{array}$ & $\begin{array}{l}\text { Tous les partenaires, chacun en } \\
\text { fonction de ses compétences }\end{array}$ \\
\hline $\begin{array}{l}\text { Concevoir et proposer des } \\
\text { services pédagogiques en ligne } \\
\text { faisant appel aux compétences } \\
\text { respectives des partenaires en } \\
\text { matière d'ingénierie de } \\
\text { formation }\end{array}$ & $\begin{array}{c}\text { Élaborer le dispositif de formation } \\
\text { pour chaque diplôme faisant appel } \\
\text { à une ingénierie de formation } \\
\text { spécifique }\end{array}$ & $\begin{array}{l}\text { Tous les partenaires, chacun en } \\
\text { fonction de ses compétences }\end{array}$ \\
\hline Communiquer et diffuser & $\begin{array}{l}\text { Promouvoir le campus numérique, } \\
\text { tant en France qu'à l'international }\end{array}$ & $\begin{array}{l}\text { Tous les partenaires, chacun en } \\
\text { fonction de ses compétences }\end{array}$ \\
\hline
\end{tabular}

Note. Les domaines identifiés dans ce tableau sont expressément nommés dans la première convention FORSE signée le 10 avril 2003.

Les directions des établissements partenaires interviennent parfois sur certaines orientations du projet, notamment celles des recrutements en personnel qui s'avèrent importantes dans le travail quotidien de l'équipe projet du campus. Deux profils ont été retenus: les "coordonnateurs de formation " qui collaborent avec les équipes pédagogiques, plus particulièrement avec les responsables des diplômes, et qui travaillent chaque jour au contact des étudiants en animant la plateforme; les « administrateurs de plateforme secondaires » dans les universités (le CNED restant la référence en termes d'administration de plateforme) administrent les plateformes et participent à la médiatisation des ressources du campus. C'est de la qualité de ce travail d'équipe que dépend la solidité d'un projet et de l'engagement ou du désengagement de l'ensemble de ses membres et non, comme l'indiquait Fichez (2007, p.11) : « malgré sa relative durée, FORSE reste encore aujourd'hui un dispositif que l'on peut qualifier de "dépendant de ses porteurs" par opposition aux dispositifs institutionnels ». En effet, près de quinze ans plus tard, nous remarquons que les porteurs de projet du départ ont su, à l'inverse, favoriser l'émergence d'un écosystème à partir du projet initial, dans lequel de nombreux acteurs s'y retrouvent (qu'ils soient enseignantschercheurs, personnels administratifs ou techniciens). Cela signifie qu'ils y trouvent des raisons de s'y 
engager et des satisfactions tant personnelles que professionnelles (apprendre, se développer, échanger, découvrir, se faire reconnaître). Ainsi, sans jeu de mots, ce campus tient sa force de la capacité des équipes qui l'animent à savoir intégrer, voire à savoir transmettre et à former en contexte de travail les nouveaux acteurs qui intègrent ce campus (enseignants-chercheurs, tuteurs, coordonnateurs, administrateurs, animateurs, personnels administratifs, techniciens, etc.) aux spécificités de la distance et de ce dispositif.

\section{Conclusion}

Par conséquent, nous retiendrons que la pérennité du campus FORSE tient à la conjonction de plusieurs facteurs de départ qui ont sans doute facilité le succès du projet : 1) la filiation directe entre les formations présentielles de Rouen et de Lyon 2, et celles délivrées à distance; 2) certains contenus qui existaient déjà, mais qu'il a fallu médiatiser; 3 ) le partenariat entre les membres du consortium ait antérieur à la réponse à l'appel à projets et des pratiques collaboratives déjà engagées. Ce sont donc sans doute des modes de management qui ont été décidés en fonction de la proximité déjà existante entre les acteurs de chacun des partenaires. Cela a permis la création d'un espace de réflexion et d'action intégré à la culture universitaire et de cet écosystème social dynamique. De même, le modèle économique du campus articule une production de supports de formation à faibles coûts fixes pour le public important accueilli en licence, dont le cadre pédagogique demeure transmissif dans une approche traditionnelle du premier cycle universitaire. À l'inverse, des modèles collaboratifs plus coûteux et qui demandent parfois aux enseignants d'évoluer dans leurs pratiques pédagogiques sont retenus pour les masters.

Ainsi, malgré la première impression qui donne à penser qu'une dynamique industrielle existe au sein du cursus FORSE, la division du travail est restée assez proche des schémas universitaires traditionnels. Ici, nous sommes persuadés que si le campus FORSE a tenu ses promesses, c'est surtout à partir d'une base constituée de valeurs communes et du soutien politique des gouvernances qui se sont succédé. Voici un autre point non négligeable : le souci de l'innovation pédagogique ou de l'anticipation d'un environnement en cours de transformation, tant du point de vue technopédagogique que financier. Nous pouvons par conséquent affirmer que le campus FORSE a su évoluer en maintenant une avance sur les mutations technopédagogiques attendues de son temps. Nous en voulons pour preuve l'introduction des webinaires il y a près de dix ans (Verquin Savarieau et Daguet, 2012) et la recherche d'une réponse aux nécessités du moment en s'adaptant et en restant à l'écoute des besoins des publics étudiants de plus en plus hétérogènes par des formes d'accompagnement multiples et variées (Verquin Savarieau et al., 2019). La pérennité du campus numérique FORSE semble en effet liée à deux aspects concomitants. Le premier concerne l'attachement aux missions mêmes de l'université (permettre l'accès à la formation au plus grand nombre). Si ce principe peut être compris par l'éducation tout au long de la vie, il est aussi une mission même de l'université, ce qui n'est pas sans créer des controverses entre une volonté de massification de la formation, les moyens alloués et la personnalisation nécessaire à la réussite dans les apprentissages, clé de la réussite en formation à distance. Cette controverse semble pouvoir être dépassée lorsque les acteurs et le politique poursuivent un but commun lié à des valeurs communes et non uniquement à des normes. Le deuxième aspect concerne les transformations opérées, au fil des années, par un processus d'acculturation par l'ensemble des acteurs jusqu'au politique, que cela soit dans l'appropriation des technologies, la reconnaissance de l'activité d'enseignement à distance à l'université ou les scénarios pédagogiques davantage orientés sur une activité collaborative. Au-delà des modifications mêmes, il est intéressant de comprendre la modification des propriétés structurantes qui assurent la pérennité du campus numérique, des acteurs jusqu'au politique et aux textes règlementaires.

Au final, si nous mettons la technologie de côté pour éviter un débat sans fin entre les technophiles et les technophobes et surtout pour ne pas en faire un mythe, le campus numérique étudié a dû dépasser les 
controverses du déterminisme technologique pour trouver la confiance et la reconnaissance institutionnelle afin que la formation à distance ne soit pas perçue comme un supplément de l'offre de formation universitaire tout comme l'activité de l'enseignant-chercheur à distance, mais en devienne une partie intégrante. Cette modification a été essentielle et a permis d'augmenter l'investissement en travail des enseignants-chercheurs ainsi que leurs appropriations pédagogiques de la distance, tout comme des environnements de travail retenus. Une autre spécificité concerne le rôle indispensable de la recherche, tant pour favoriser des innovations technopédagogiques que pour évaluer leurs effets, permettant un dialogue objectif avec le politique. Si la diffusion et la dissémination de la recherche peuvent être un processus social long, elles sont essentielles dans un environnement universitaire sensible à des résultats permettant d'améliorer tout dispositif qui répond à des attentes sociales.

Le rôle des enseignants-chercheurs est ainsi primordial pour produire des savoirs praxéologiques et axiologiques au sein d'une institution où ils ont une reconnaissance par leur activité scientifique, leur statut et fonctions institutionnelles. Enfin, un dernier enjeu tient à la possibilité d'anticiper l'évolution de la société et des institutions. Ce travail incessant nécessite certainement des enseignants-chercheurs chevronnés et militant pour la démocratisation des savoirs sans rompre avec la liberté qui spécifie son activité. La tâche est lourde, harassante, souvent conflictuelle dans une université où la formation en présentiel est le fondement même de cette institution, où l'enseignement magistral est constitutif d'une genèse identitaire solidement ancrée. C'est donc avec du temps, de la patience, un turn-over des responsabilités assurées dans la continuité impliquant aussi des recrutements qu'il est possible de pérenniser un campus numérique en modifiant, au fil des temps, certaines de ses propriétés curriculaires, financières, organisationnelles, productives, se percolant en ce qui concerne l'université qui elle-même a su tirer bénéfice de ce campus en fonction de ses propres contraintes : nouveau public de formation, souci de la rentabilité des formations, adéquation aux réformes de la formation continue, etc. Jusqu'à présent, ce campus a pu anticiper certaines attentes et faire face à de nombreux changements explicitant, pour partie, sa pérennité au prix de l'investissement constant de ses acteurs, notamment des responsables pédagogiques spécialistes en technologies de l'éducation. Cependant, il faut bien le souligner, lors de chaque renouvellement de convention, ce campus est remis en question, puisqu'il faut à nouveau trouver un équilibre entre les propriétés de ce campus et celles de l'ensemble des partenaires et de leurs enjeux propres. II n'est pas certain que les valeurs universelles l'emportent sur le gain direct obtenu par ceux qui s'y investissent ou qui en attendent des retombées quantitatives dans la course effrénée au nombre d'inscrits et au gain de productivité.

\section{Liste de références}

Akrich, M., Callon, M. et Latour B. (dir.). (2006). Sociologie de la traduction : textes fondateurs. Paris, France : Presses des Mines.

Albero, B. et Simonian S. (2019). Les conditions d'un dialogue majorant entre le politique et le pédagogique. Le cas du Campus numérique FORSE. Dans A. Jézégou (dir.), Traité de la e-formation des adultes (p. 25-48). Bruxelles : De Boeck.

Albero, B. et Brassac, C. (2013). Une approche praxéologique de la connaissance dans le domaine de la formation. Éléments pour un cadre théorique. Revue française de pédagogie, 184(3), 105-119.

Albero, B., Linard, M. et Robin, J.-Y. (2009). Petite fabrique de l'innovation ordinaire à l'université. Quatre Parcours de pionniers. Paris : L'Harmattan.

Albero, B. et Thibault, F. (2006). Enquête auprès des universités françaises. Dans Les universités européennes à l'heure du e-learning : regard sur la Finlande, I'ltalie et la France (p. 61-98). Paris : Agence de Mutualisation des Universités et Établissements.

Annoot, E. (2014). Postface. Dans G. Lameul, et C. Loisy (dir.). La pédagogie universitaire à l'heure du numérique, questionnement et éclairage de la recherche (p. 221-226). Bruxelles : De Boeck Université. 
Ardouin, T. (2007). Ce qui compte dans les formations à distance, c'est la présence ! Le cas du master ICF. Dans J. Wallet (dir.), Le Campus numérique FORSE : analyses et témoignages (p. 17-29). Rouen : Presses universitaires de Rouen et du Havre.

Aubert, G. (2001) Editorial. CNED Canal education, $n^{\circ} 20$, p.3.

Averous, M. et Touzot, G. (2002). Rapport Campus numériques. Enjeux et perspectives pour la formation ouverte et à distance. https://eduscol.education.fr/chrgt/CNenjeux.pdf

Barbot, M.-J. et Combès, Y. (2006). Penser le changement de paradigme éducatif lié aux TIC. Éducation permanente, 4(169), 133-152.

Baron, G.-L. (2010). Quelles évolutions des professionnalités dans le contexte de l'enseignement supérieur en ligne? Distances et savoirs, 8(2), 193-205.

Bédard, D. et Béchard, J.-P. (2009). Innover dans l'enseignement supérieur. Paris : PUF.

Béziat, J. (2012). Formateur en ligne : vers un modèle d'action. Distances et médiations des savoirs, 2012-213(1). https://doi.org/10.4000/dms.116

Callon, M. (1986). Éléments pour une sociologie de la traduction. La domestication des coquilles Saint-Jacques et des marins dans la baie de Saint-Brieuc. L'année sociologique, 36, 170-208.

Commission européenne. (2001). Réaliser un espace européen de l'éducation et de formation tout au long de la vie. ${ }^{*} \mathrm{COM} / 2001 / 0678$ final * : https://eur-lex.europa.eu/legal-content/FR/TXT/?uri=celex\%3A52001DC0678

Deceuninck, J. (2007). Les campus numériques en France : réalisations, dynamiques et émergences. Études de communication, numéro spécial. https://doi.org/10.4000/edc. 617

Deceuninck, J. (2005). Campus FORSE, Formation et Ressources en Sciences de l'éducation : Rapport exploratoire. Modèles économiques et enjeux organisationnels des campus numériques. Paris : MSHS. https://hal.archives-ouvertes.fr/hal-01388309/document

Denis, B. (2003). Quel rôle et quelle formation pour les tuteurs intervenant dans les dispositifs de formation à distance? Distance et savoirs, 1(1), 19-46.

Develay, M. et Godinet, H. (2007). Éléments pour une problématique du changement. Dans J. Wallet (dir.), Le Campus numérique FORSE : analyses et témoignages (p. 17-29). Rouen : Presses universitaires de Rouen et du Havre.

Eneau, J., Simonian, S. et Siméone, A. (2008). TIC et enseignement universitaire : vers une nouvelle professionnalité enseignante? Le cas du Campus FORSE à l'Université Lyon 2. Dans Actes du $5^{e}$ colloque "Questions de pédagogies dans l'enseignement supérieur - Enseigner, étudier dans le supérieur : pratiques pédagogiques et finalités éducatives » (p. 267-274), Brest. https://hal.archives-ouvertes.fr/hal-00588878/document

Engeström, Y. (2001). Expansive learning at work: toward an activity theoretical reconceptualization. Journal of Education and Work, 14, 133-156.

Fichez, E (2007). Campus numériques français : pertinence des notions de réussite ou d'échec. Études de communication, numéro spécial. https://doi.org/10.4000/edc.567

Gauttier, P. (2012) La validation des acquis de l'expérience à l'université. Revue française d'administration publique, 144(4), 1107-1119.

Glikman, V. (2002). Des cours par correspondance au e-learning: panorama des formations ouvertes et à distance. Paris : PUF.

Grevet, P (2005). Du rapport Quéré aux appels à projets campus numériques : quelques orientations économicoinstitutionnelles. Modèles économiques et enjeux organisationnels des campus numériques. https://hal.archives-ouvertes.fr/hal-01388304/document

Gérin-Lajoie, S. et Potvin, C. (2011). Évolution de la formation à distance dans une université bimodale. Distances et savoirs, 3(3), 349-374. https://www.cairn.info/revue-distances-et-savoirs-2011-3-page-349.htm

Houssaye, J. (2007). Le MARDIF. Dans J. Wallet (dir.), Le Campus numérique FORSE : analyses et témoignages (p. 105-111). Rouen : Presses universitaires de Rouen et du Havre.

Kim, S.-M. et Verrier, C. (2009). Le plaisir d'apprendre en ligne à l'université. Implication et pédagogie. Bruxelles : De Boeck, Perspectives en éducation \& formation. 
Jacquinot, G. et Fichez, É. (2008). L'université et les TIC: Chronique d'une innovation annoncée. De Boeck Supérieur.

Jacquinot, G., (1993). Apprivoiser la distance et supprimer l'absence ou les défis de la formation à distance. Revue Française de Pédagogie, 102, 55-68.

Jacquinot, G. (1985). L'école devant les écrans. Paris : ESF.

Lameul, G. et Loisy, C. (dir.). (2012). La pédagogie universitaire à l'heure du numérique, questionnement et éclairage de la recherche. Bruxelles : De Boeck Université.

Lapalme, M.-E. (2003) Portrait de l'université virtuelle renouvelée et de ses enjeux pédagogiques. Cahiers de recherche OIPG, $n^{\circ}$ 2003-00. https://tinyurl.com/k3ntmijk

Latour, B. (1992). Aramis ou l'amour des techniques. Paris, France : La Découverte.

Linard, M. (2003). Campus numérique FORSE. Rapport scientifique. https://edutice.archives-ouvertes.fr/edutice-00137546

Linard, M. (2001). Concevoir des environnements pour apprendre : l'activité humaine, cadre organisateur de l'interactivité technique. Sciences et Techniques Educatives, 8(3-4), 211-238.

Linard, M. (1990). Des machines et des hommes, Apprendre avec les nouvelles technologies. Éditions universitaires.

Lison, C. et Jutras, F. (2014). Innover à l'université : Penser les situations d'enseignement pour soutenir l'apprentissage. Revue internationale de pédagogie de l'enseignement supérieur, 30(1). http://journals.openedition.org/ripes/769

Miguet, M. (2011). Depuis une décennie, la FAD dans les universités françaises : Au croisement des discours de modernisation et du terrain. Distances et savoirs, 9(3), 331-347. https://www.cairn.info/revue-distances-et-savoirs-2011-3-page-331.htm

Lison, C. et Jutras, F. (2014). Innover à l'université : penser les situations d'enseignement pour soutenir l'apprentissage. Revue internationale de pédagogie de l'enseignement supérieur, 30(1). https://doi.org/10.4000/ripes.769

Mayen, P. et Métral, J.-F. (2008). Compétences et validation des acquis de l'expérience. Formation emploi, 1(101), 183-197. https://www.cairn.info/revue-formation-emploi-2008-1-page-183.htm

Menger, P.-M. (2009). Le travail créateur. S'accomplir dans l'incertain. Paris, Du Seuil.

Mœglin, P. (2016). Introduction : La question de l'industrialisation de l'éducation. Dans : Pierre Mœglin éd., Industrialiser l'éducation : Anthologie commentée (1913-2012) (p. 9-73). Saint-Denis, France: Presses universitaires de Vincennes. https://www.puv-editions.fr/media/ouvr pdf/635 Intro industrialiser.pdf

Mœglin, P. et Thibault, F. (2009). Universités et ressources numériques : une affaire entre acteurs publics, Quaderni, 69, printemps. https://doi.org/10.4000/quaderni.311

Paivandi, S. (2009). L'enseignement à distance : un facteur de changement à l'université. Dans : S. M. Kim et C. Verrier (dir.), Le plaisir d'apprendre en ligne à l'université: Implication et pédagogie (p. 177-188). Louvain-la-Neuve, Belgique : De Boeck Supérieur.

Pinte G. (2008). VAE et stratégie pour la fonction RH des entreprises : Quelles contribution des services de formation continue des universités? Dans L. Ben Moussi Le Gall (dir.), Validation des Acquis de l'Expérience. Retour d'expériences à l'université (p. 143-156). Paris : L'Harmattan.

Porot-Viaouet, M. (2007). Répartition géographique des étudiants rouennais. Dans J. Wallet (dir.), Le Campus numérique FORSE : analyses et témoignages (p. 77-81). Rouen : Presses universitaires de Rouen et du Havre.

Quéré, M. (1994, juin). Vers un enseignement sur mesure. Ministère de l'Enseignement supérieur et de la Recherche, Direction générale des enseignements supérieurs, France.

Simonian, S. et Verquin Savarieau, B. (2016). Introduction au dossier « Mutations de l'accompagnement dans les formations en ligne ». Distances et médiations des savoirs, 13. https://doi.org/10.4000/dms.1335

Simonian, S. (2014). L'affordance socioculturelle : une approche éco-anthropocentrée des objets techniques. Le cas des Environnements Numériques d'Apprentissage. (Note de Synthèse de l'Habilitation à Diriger les Recherches). Université Rennes 2, France.

Thibault, F. (2007). Campus numérique : archéologie d'une initiative ministérielle. Études de communication, numéro spécial. https://doi.org/10.4000/edc.546 
Thibault, F. (2002). De l'université virtuelle au campus numérique : simple effet de traduction ou changement de paradigme. Actes du premier colloque franco-mexicain de sciences de la communication. Mexico, Mexique. CERIMES.

Thibault, F. (2001). Campus numériques français. Un contexte international. CNED Canal éducation, 20, p. 4-5.

Verquin Savarieau, B. et Daguet, H. (2020). L'intégration de la classe virtuelle synchrone à l'université, un levier de transformation de la professionnalité enseignante? Médiations et médiations, 3. https://doi.org/10.52358/mm.vi3.109

Verquin Savarieau, B., Annoot, E., Daguet, H., Rinaudo, J.-L. (2019). La notion de "partenariat » et son actualité dans la formation à distance. Retour d'expérience du campus numérique FORSE. Colloque international Education 4.1!, 18 janvier 2019, CNED- EIFAD. https://tinyurl.com/5bmau2de

Verquin Savarieau, B. (2013). La validation des acquis professionnels une pratique de professionnalisation? Les dossiers des sciences de l'éducation, 30. https://doi.org/10.4000/dse.256

Verquin Savarieau, B. et Daguet, H. (2012). L'introduction des classes virtuelles synchrones, un moyen de renforcer la qualité de l'accompagnement en formation d'adultes? frantice.net, 6. http://www.frantice.net/index.php?id=710

Wallet, J. (2007). Le campus numérique FORSE : analyses et témoignages. Rouen: PURH.

Wittorski, R. (2012). La professionnalisation de l'offre de formation universitaire : quelques spécificités. Revue internationale de pédagogie de l'enseignement supérieur, 28(1). http://ripes.revues.org/580 